\title{
INTRACRANIAL HEMORRHAGE IN A CHILD OF HEMOPHILIA: A CASE REPORT
}

Pankaj Chaudhari1 ${ }^{1}$ Ashutosh Chitnis², Saurabh Pawar³, Akshay Gursale4, Ishan Pranay5

\section{HOW TO CITE THIS ARTICLE:}

Pankaj Chaudhari, Ashutosh Chitnis, Saurabh Pawar, Akshay Gursale, Ishan Pranay. "Intracranial Hemorrhage in a Child of Hemophilia: A Case Report". Journal of Evolution of Medical and Dental Sciences 2015; Vol. 4, Issue 79, October 01; Page: 13913-13918, DOI: 10.14260/jemds/2015/1982

ABSTRACT: Intracranial hemorrhage (ICH) in children with hemophilia is not a commonly occurring event. Retrospective studies performed estimate the incidence of ICH to be 3.4\%-4.0\%. Signs of ICH in children clinically are difficult to assess and often result in a delay in the diagnosis. The timely diagnosis of ICH is critical for the early and intensive factor replacement therapy that the patient needs. We report a case of ICH in a child with severe hemophilia A with no family history.

INTRODUCTION: Intracranial hemorrhage (ICH) is the abnormal accumulation of blood in the cranial vault that may occur within the brain parenchyma or in the surrounding meningeal spaces. ${ }^{11]}$ ICH is the most feared complication of hemophilia; 20\% of children with ICH die. Timely diagnosis and prompt initiation of therapy with ICH are critical, even though the clinical signs of ICH in children are difficult to assess.

CASE REPORT: A male child 6 years of age was brought with complaints of fever and vomiting since 3 days. The fever was moderate to high grade in nature, intermittent and relieved on medication. The child had multiple episodes of vomiting containing food particles initially then clear fluid. The vomitus was non-bilious, non-projectile and not blood stained.

Since 2 days the child was lethargic and was responding only to the stimulus. He had reduced oral intake. Since the time of illness the child is not even sitting without support.

At 5 years of age the child fell down and got fracture at left knee joint for which he was operated. Since then the child is not walking and there was no movement in left lower limb. The child was diagnosed as a Hemophilic at two years of age. He was born out of the non-consanguinous marriage and was the first order by birth. The parents were of low socio-economic status. The child was delivered as full term normally at Hospital. The child was immunized fully till date.

At the time of examination the child was afebrile, drowsy, lethargic and was aroused after painful stimuli. Tone was normal in both upper limbs and in right lower limb. Left lower limb showed decreased tone. Power was 4/5 in both upper limbs and right lower limb and 2/5 in left lower limb. Neck stiffness was present. Blurred optic disc margins were seen bilaterally on fundus examination.

CBC report showed $\mathrm{Hb} 10.4$, TLC $=10500$, DLC=85/12/03/00, Platelets $=834000, \mathrm{MCV}=77.6$, $\mathrm{MCH}=25.1, \mathrm{MCHC}=32.2$ and $\mathrm{PCV}=32.3$. FACTOR VIIIC was $4.5 \%$ of the normal pooled plasma (NR -50 $150 \%$ of NPP).

CT scan and Magnetic Resonance Imaging (MRI) of the brain revealed multiple hematomas in left frontal and high parietal lobe, in right cerebellar hemisphere, downward tonsillar herniation and indentation of the posterior eyeball suggestive of raised intracranial tension. 


\section{CASE REPORT}
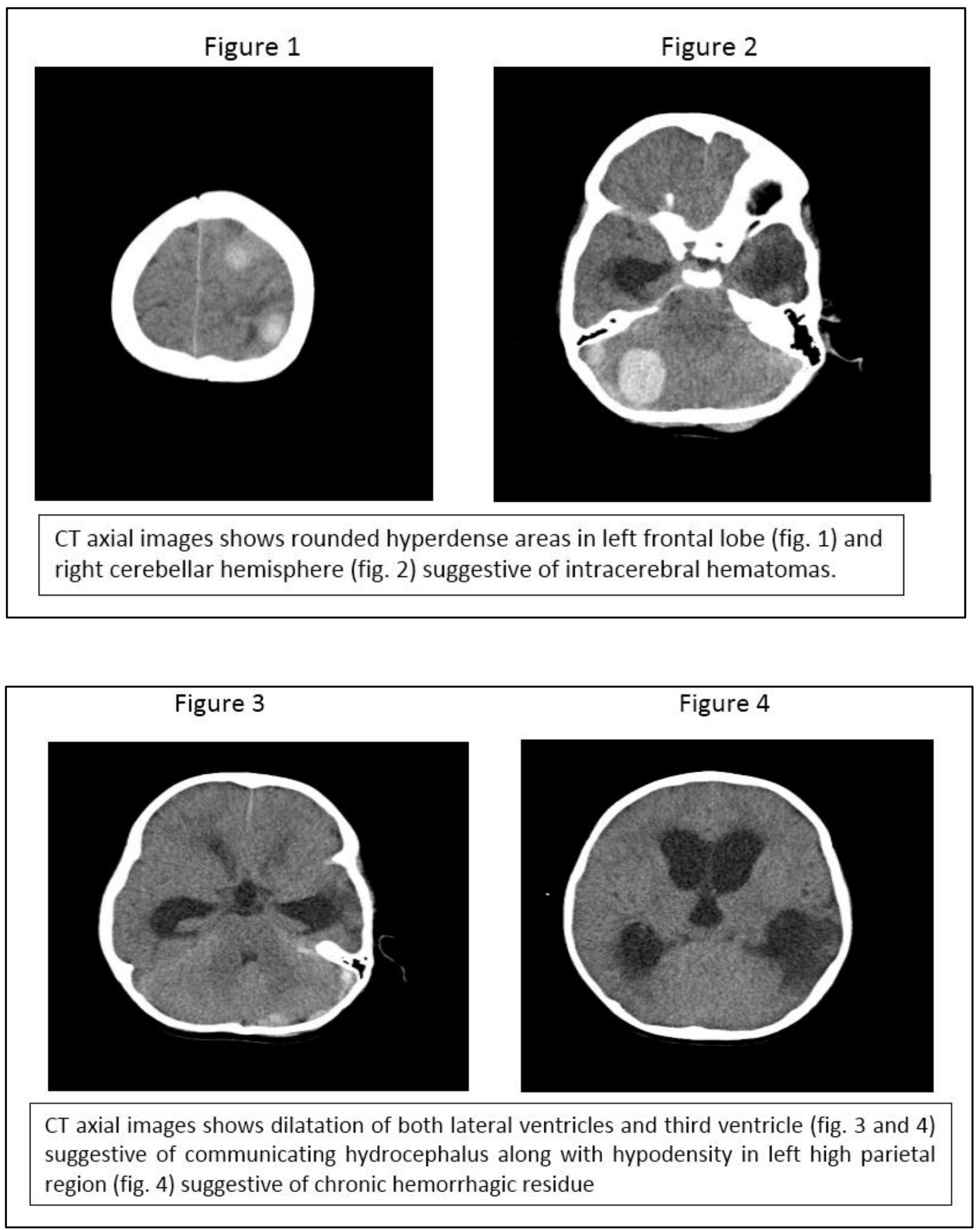


\section{CASE REPORT}
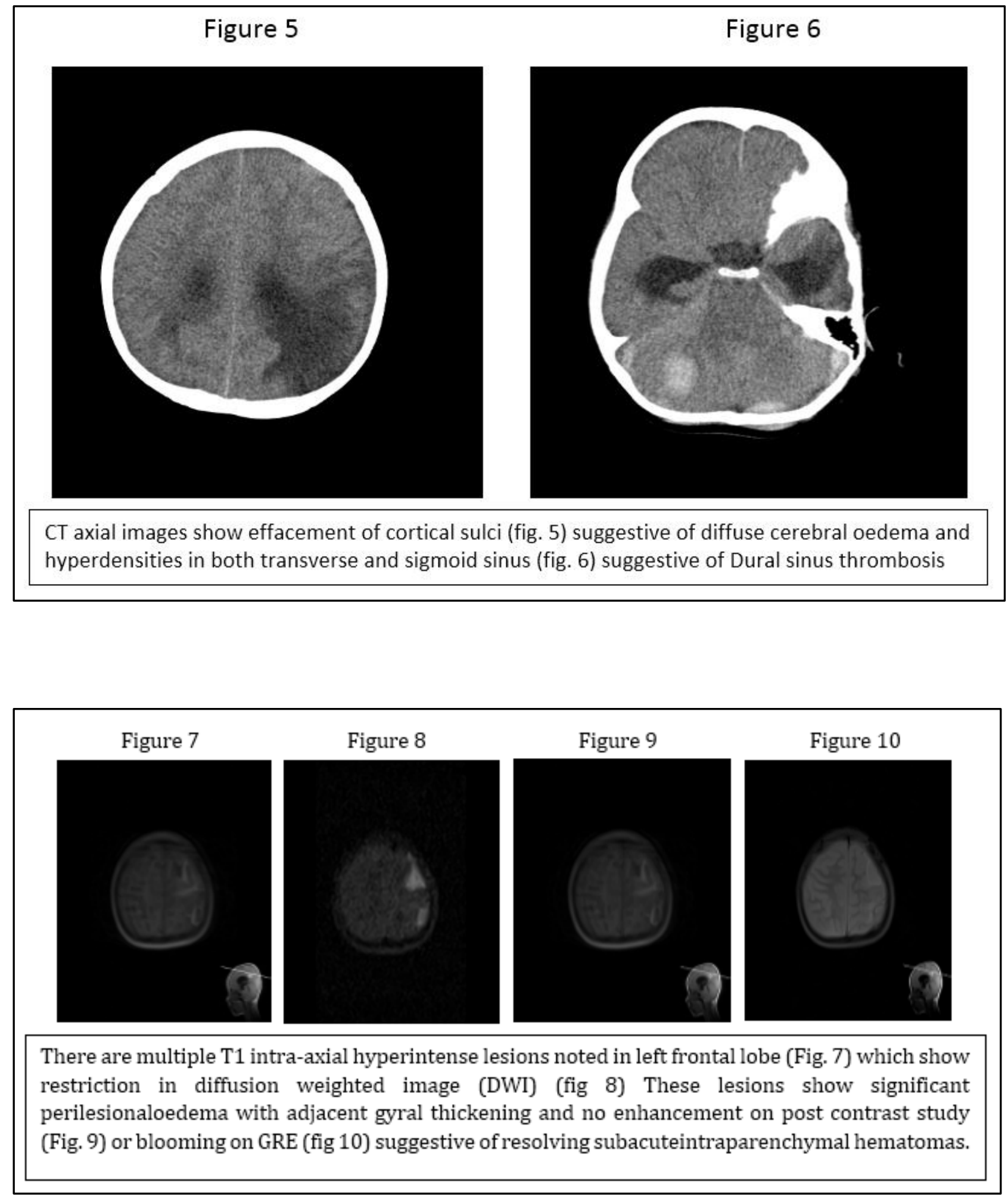


\section{CASE REPORT}
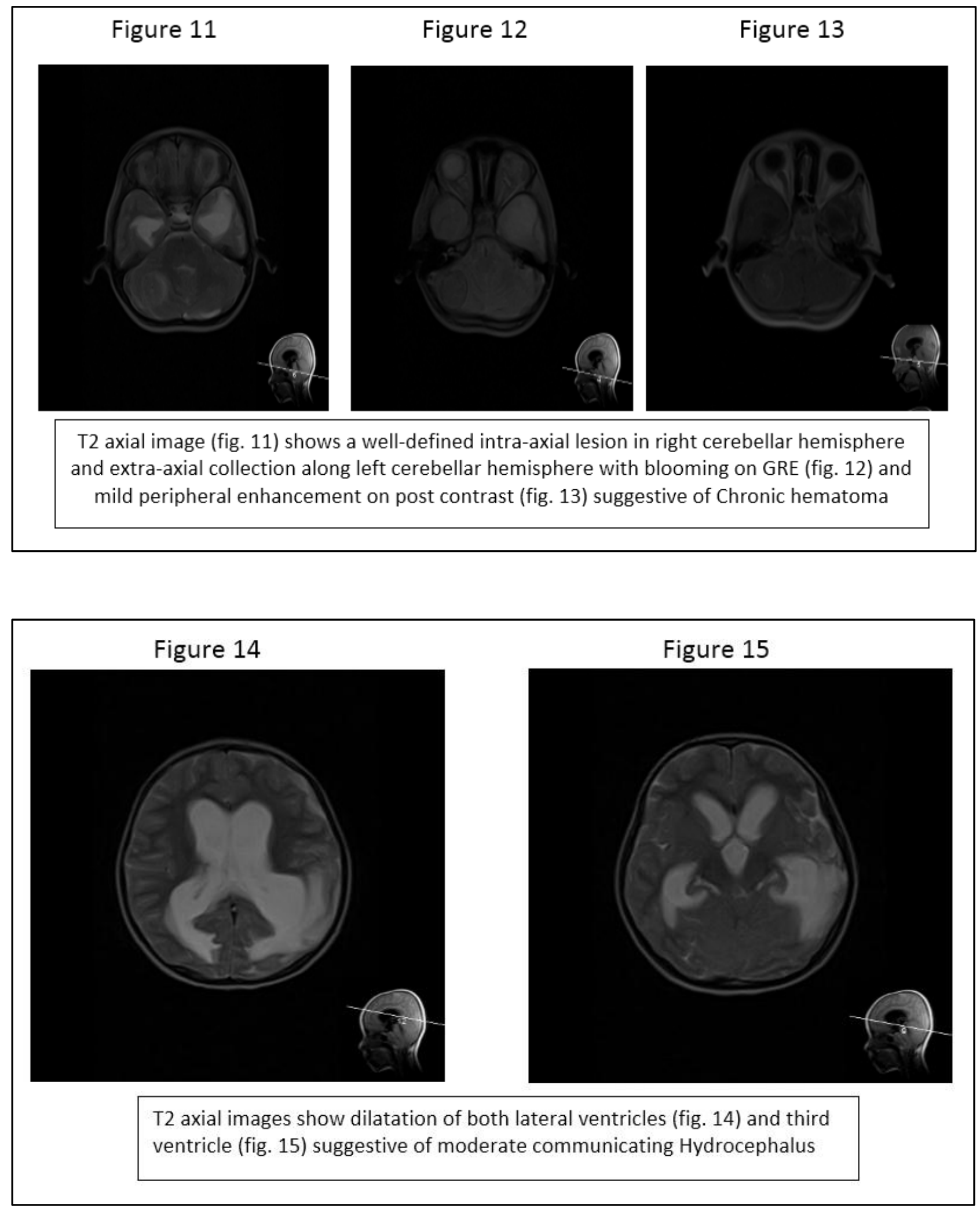


\section{CASE REPORT}

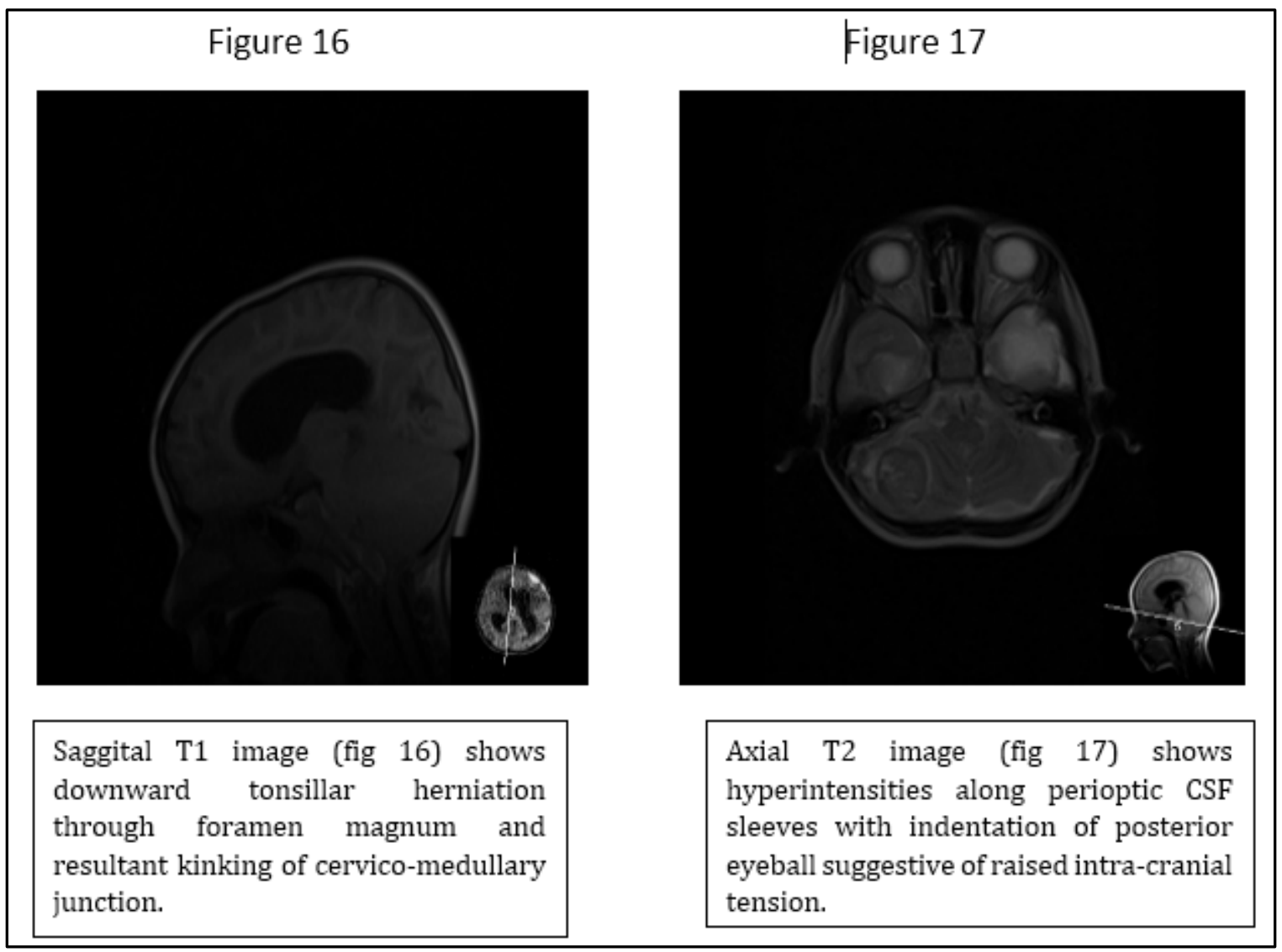

The child was started on recombinant anti-hemophilic Factor VIII concentrate infusion for a total of 10 days. His irritability resolved, he was tracking visually and smiling, and his physical examination remained unremarkable. A follow-up CT scan revealed a stable hemorrhage with no increase in size. The patient was discharged with further regular infusions prescribed.

DISCUSSION: Although ICH is associated with increased morbidity and mortality, delayed diagnosis occurs for a variety of reasons, including vague symptoms-such as the irritability and fever in this case-that may be confused with other conditions or a lack of awareness about hemophilia.[2]

The outcomes of people with hemophilia who experienced an ICH improved significantly in the 1970s because of the introduction of more sophisticated imaging modalities, such as CT and MRI.[3] With the regular use of factor concentrates and prophylaxis (The use of infusions on a schedule to prevent bleeding), the mortality rates for ICH declined but remain a considerable challenge (20\%-30\%).[4] Additionally, the risks of neurologic sequelae are substantial. Common neurologic sequelae include seizures (63\% of cases), psychomotor (59\%) and statomotor retardation (52\%), and cerebral palsy (45\%).[5]

Studies report that children with hemophilia who experienced ICH are significantly more likely to have neurocognitive, academic, behavioral, adaptive, and motor functioning deficiencies. These findings strongly suggest that long-term follow-up of children with ICH, including neuropsychological assessment and intervention, is critical.[6]

Because of ICH's elevated risks and high mortality rate, it is a true medical emergency and should be treated presumptively prior to any evaluation. 
CONCLUSIONS: Even the subtle signs associated with ICH should trigger the suspicion of an episode, and treatment should be given preemptively. In any case of suspected ICH in neonates or children with hemophilia, contact with a regional hemophilia treatment center immediately or prior to discharge from the emergency department or the hospital can be lifesaving. ICH should be managed in an experienced center; however, treatment should not be delayed until after transfer to the treatment center. Finally, comprehensive rehabilitation and close long-term follow-up with the hemophilia treatment center are essential to an optimal recovery.

\section{REFERENCES:}

1. Ljung R, Lindgren AC, Petrini P, Tengborn L. Normal vaginal delivery is to be recommended for haemophilia carrier gravidae. Acta Paediatr. 1994 Jun; 83(6):609-611.

2. Kulkarni R, Lusher JM. Intracranial and extracranial hemorrhages in newborns with hemophilia: a review of the literature. J Pediatr Hematol Oncol. 1999 Jul-Aug; 21(4):289-295.

3. Mishra P, Naithani R, Dolai T, et al. Intracranial haemorrhage in patients with congenital haemostatic defects. Haemophilia. 2008 Sep; 14(5):952-955. Epub 2008 Jul 14.

4. Quinones-Hinojosa A, Gulati M, Singh V, Lawton MT. Spontaneous intracerebral hemorrhage due to coagulation disorders. Neurosurg Focus. 2003 Oct 15; 15(4):E3.

5. Smith ML, Bauman JA, Grady MS. Neurosurgery. In: Brunicardi FC, Andersen DK, Billiar TR, et al, eds. Schwartz's Principles of Surgery. 9th ed. New York, NY: McGraw-Hill; 2010:1515-1556.

6. Tarantino MD, Gupta SL, Brusky RM. The incidence and outcome of intracranial haemorrhage in newborns with haemophilia: analysis of the Nationwide Inpatient Sample database. Haemophilia. 2007 Jul; 13(4):380-382.

\section{AUTHORS: \\ 1. Pankaj Chaudhari \\ 2. Ashutosh Chitnis \\ 3. Saurabh Pawar \\ 4. Akshay Gursale \\ 5. Ishan Pranay}

\section{PARTICULARS OF CONTRIBUTORS:}

1. $2^{\text {nd }}$ Year Junior Resident, Department of Radio-diagnosis, MGM Medical College and Hospital, Kamothe, Navi Mumbai.

2. Associate Professor, Department of Radiodiagnosis, MGM Medical College and Hospital, Kamothe, Navi Mumbai.

3. $2^{\text {nd }}$ Year Junior Resident, Department of Radio-diagnosis, MGM Medical College and Hospital, Kamothe, Navi Mumbai.

FINANCIAL OR OTHER COMPETING INTERESTS: None
4. $3^{\text {rd }}$ Year Junior Resident, Department of Radio-diagnosis, MGM Medical College and Hospital, Kamothe, Navi Mumbai.

5. $2^{\text {nd }}$ year Junior Resident, Department of Radio-diagnosis, MGM Medical College and Hospital, Kamothe, Navi Mumbai.

\section{NAME ADDRESS EMAIL ID OF THE CORRESPONDING AUTHOR:}

Pankaj Chaudhari, \#304, Sadhana Darshan Chs, Kopar Road, Near Kopar Station, Dombivali-421202, (West), Thane, Maharashtra.

E-mail: drpankajsc@yahoo.co.in

Date of Submission: 03/07/2015. Date of Peer Review: 04/07/2015. Date of Acceptance: 23/07/2015. Date of Publishing: 30/07/2015. 